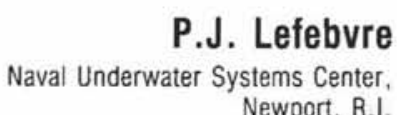

Newport, R.I.

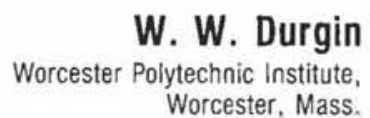

\section{A Transient Electromagnetic Flowmeter and Calibration Facility}

An electromagnetic flowmeter was developed to measure transient flows at a dato rate of $60 \mathrm{~Hz}$. The approach taken was to develop suitable electronics to replace the electronics of a commercially available electromagnetic flowmeter normally used for steady-state operation. Use of the commercially available flowmeter body, which includes the magnetic coils, core, and signal electrodes, provided a relatively economical means of fabricating the transient flowmeter. A transient flow calibration facility consisting of a free-falling water column was also designed and built. Results of the calibrations are presented and show that the flowmeter can accurately measure transient flows up to the maximum observed acceleration of approximately $1 \mathrm{~g}$.

\section{Introduction}

In the study of transient pipe flows, a need exists for the accurate measurement of the mean volumetric flow rate as a function of time. To date, very few flowmeters have been built that have the capability to measure transient flows. In addition, to the authors' knowledge, there are no commercially available flowmeters that can accurately measure transient flow rates at a high data rate.

Many of the existing flowmeters used for steady-state applications are inherently not applicable to transient flow measurements. For example, turbine meters, even though they have a fast signal response time and consequently provide output at an apparent high data rate, are momentum devices and the meter calibration is inherently a function of not only velocity profile but also rate of change of flow rate.

Head type meters, such as orifice plates, are dependent on the response of the pressure transducer(s) used in the differential pressure measurement. In addition, the flow field in the vicinity of the orifice can change considerably under high flow transients leading to changes in the orifice's calibration.

A few special-purpose meters have been developed over the past 25 years that have had some transient flow measurement capabilities. In 1950, Arnold [(1)] described an electromagnetic flowmeter for small flow rates that responded to very short transients of 0.001 second in duration. Since a circular cross section proved unsatisfactory for the design, a small rectangular cross section of $0.48 \times 1.58 \mathrm{~cm}$ was chosen.

In 1960, Iwanicki and Fontaine [(2)] described an electromagnetic flowmeter, which was also designed to respond to very short transients of low flow rates. The meter, with a $1.2-\mathrm{cm}$ diameter, could not be operated for more than a few seconds due to polarization of the flowing liquid at the signal electrodes. In each of the references, it was shown that the meters responded to large flow transients. Unfortunately, the facilities used to validate meter performance could not provide information on the accuracy of the flowmeter output.

Contributed by the Fluids Engineering Division for publication in the JoURNAL of FluIDS EngINEERING. Manuscript received by the Fluids Engineering Division April 4, 1988.
To fulfill the need for accurate transient flow measurement during future experimental studies, the Naval Underwater Systems Center (NUSC) developed the transient flowmeter discussed herein. Criteria for this meter included accurate measurement of the mean volumetric flowrate (cross-sectional averaged velocity) with the meter being insensitive to changes in velocity profile, especially varying axi-symmetric profiles.

The resulting flowmeter is based on electromagnetic flowmeter principles and has the capability to accurately measure transient flows with a data rate of at least $60 \mathrm{~Hz}$. The meter has a $5-\mathrm{cm}$ diameter and can be used, with one common meter calibration factor, for both steady-state flows and for transient flows of either short or long duration. It also can operate accurately over a 30:1 flow rate range.

To evaluate the flowmeter's accuracy, a transient calibration facility was designed by NUSC and the Worcester Polytechnic Institute (WPI) and fabricated at WPI.

This paper describes the flowmeter, the calibration facility and also presents data on the flowmeter calibration.

\section{Flowmeter Description}

Electromagnetic technology was chosen as the basis for the transient flowmeter because of inherent fast response to transients and the general insensitivity of electromagnetic flowmeters to changes in velocity profile.

By using a weighting function, Shercliff $[(3)]$ showed mathematically that the signal generated at the electrodes represents the average velocity for axisymmetric profiles. Reinhold [(4)] presented experimental data that showed errors of less than 0.5 percent when large unsymmetric flow disturbances were placed only five pipe diameters upstream of an electromagnetic flowmeter. Typical disturbances were a concentric orifice plate with 50 percent blockage and a segmental orifice with a 50 percent blockage. Obviously, the high performance observed is dependent on the design of the flowmeter itself, primarily the flowmeter's ability to generate a suitable magnetic field.

The electromagnetic flowmeter is based on Faraday's law of induction. As a conducting fluid passes through a magnetic 


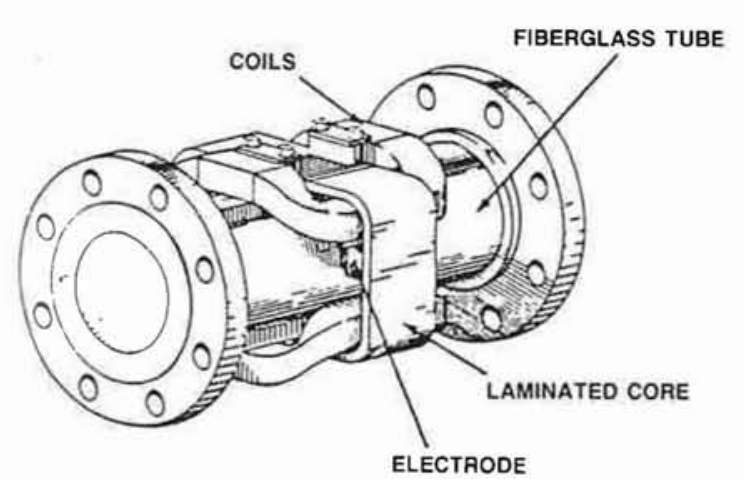

Fig. 1 Flowmeter body

field, an electric voltage is generated in a plane perpendicular to the plane of the magnetic flux vector and the conducting fluid's velocity vector. The generated potential $E$ (volts) given by Faraday's law is shown in equation (1) as

$$
E=K D B V \text {, }
$$

where $K$ is the meter's calibration factor, $D$ is the distance between signal electrodes (or meter diameter), $B$ is the magnetic llux density, and V is the cross-sectional average fluid velocity.

The approach taken in the development of the flowmeter was to utilize as much of a commercially available electromagnetic flowmeter as was feasible. The final version of he transient flowmeter, therefore, consists of the flow tube (or body) portion of a Foxboro Co. ac-powered electromagnetic llowmeter and the NUSC-designed electronics transmitter. The flow tube, as shown in Fig. 1, consists of the magnetic coils and core (which generates the magnetic flux), and the signal clectrodes mounted flush with the inner wall of a 5-cm-diameter fiberglass tube.

In operation, the coil which produces the magnetic field is powered by line voltage ( 120 volts, $60 \mathrm{hz}$ ), thereby producing a magnetic field which oscillates sinusoidally at $60 \mathrm{~Hz}$. Consequently, the signal from the electrodes is also sinusoidal at the same frequency as the magnetic field.

The electronic unit, which is essentially digital, samples the resulting sinusoidal output voltage from the signal electrodes during each cycle. The electronic unit monitors the change in voltage (flow rate) from the previous cycle and then incorporates that value into the aggregate sum to obtain the current vulue of voltage (flow rate).

Changes in the magnitude and frequency of the induced mignetic flux due to fluctuations in line voltage are compensuted for in the design. A reference coil located on the external will of the flow tube and in the vicinity of the magnetic coils monitors the instantaneous magnetic flux. By comparing the voltage from the signal electrodes to that induced in the refrence coil in a unique manner, the effect of line voltage changes arc climinated. The electronic circuits were designed to operate it frequencies up to approximately $1000 \mathrm{~Hz}$. However, at these hipher frequencies, a commercially available flowtube would he unsatisfactory; a special flowtube would be needed.

The design of the electronics includes many novel features which act together to circumvent the usual problems associated with the high noise inherent in the signal generated at the clectrodes of magnetic flowmeters. The flowmeter output provicles both an analog and a digital signal corresponding to the instintaneous flow rate. Since the magnetic field is cycled and

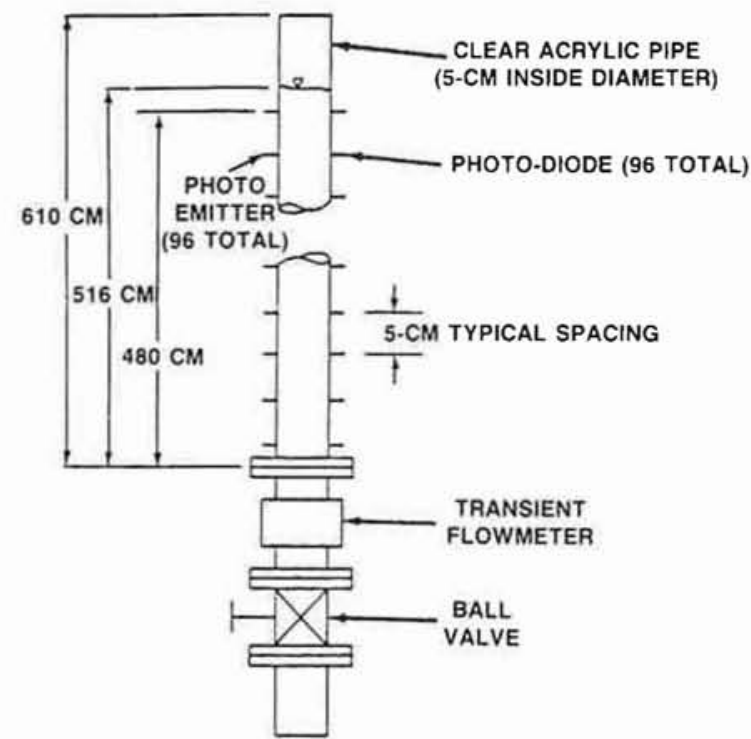

Fig. 2 Transient flow calibration facility

not maintained at a constant dc value, polarization of the fluid at the signal electrodes does not occur and the flowmeter can be operated for an indefinite period of time.

\section{Calibration Facility}

The purpose of the calibration facility at WPI was to establish the accuracy to which the flowmeter could measure transient volumetric flow rates under accelerations up to $1 \mathrm{~g}$. The general concept of the facility was to accurately measure the transient velocity of a free-falling vertical column of water to which the output from the transient flowmeter could be compared. The velocity of the water column (calibration velocity) was measured by monitoring the rate of change in elevation of the free surface of the column.

As shown in Fig. 2, the water column is contained within a clear acrylic pipe having an inside diameter of $5 \mathrm{~cm}$. The transient flowmeter was installed at the lower end of this pipe. Each test was initiated by manually opening a ball valve placed below the flowmeter. To measure the free surface velocity, a total of 96 photo-emitter/photo-diode optical pairs were distributed every $5 \mathrm{~cm}$ along the lowermost $480 \mathrm{~cm}$ of the acrylic pipe. As the surface of the water column passed a sensor station, a change in the intensity of light transmitted from the photo-emitter to the photo-diode occurred, resulting in a sharp jump in the voltage generated at the photo-diode. This change in voltage level was used to trigger a flip-flop in the facility's electronic circuitry to which all of the optical sensors were multiplexed. The analog output of this electronic system was a square wave with a varying half-period equal to the time between consecutive trigger pulses.

An IBM personal computer with a Data Translations analogto-digital data acquisition board was used to detect the trigger pulses by sampling the analog output of the electronics at a rate of $5000 \mathrm{~Hz}$. At that rate, the time that the water surface passed each of the optical sensor pairs was known to within \pm 0.0002 second.

The effective vertical distance between optical sensor pairs

Nomenclature

$A=$ cross-sectional area

$B=$ magnetic flux density

D) = meter diameter
$E=$ voltage generated at electrodes

$K=$ meter calibration factor

$L=$ effective distance between optical pairs

$$
\begin{aligned}
V= & \text { cross-sectional average fluid } \\
& \text { velocity } \\
W= & \text { weight } \\
\rho & =\text { fluid density } \\
\sigma & =\text { standard deviation }
\end{aligned}
$$




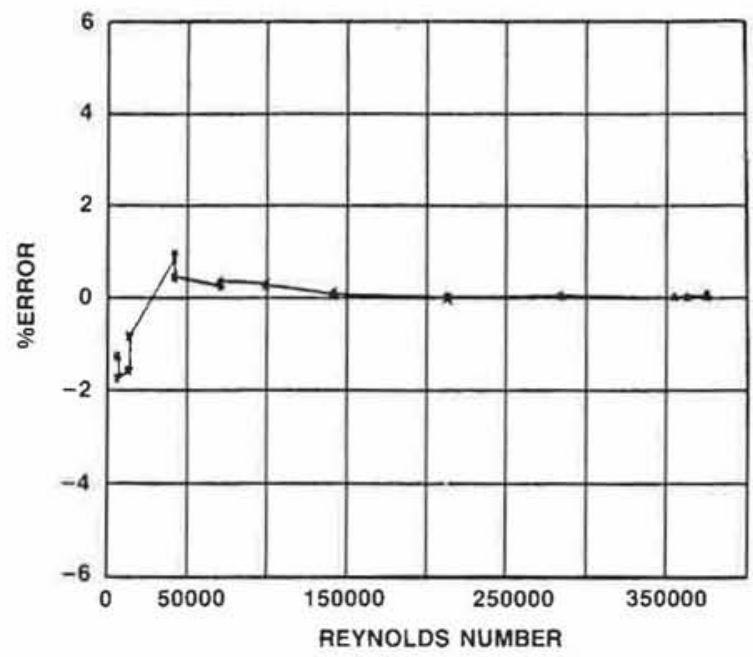

Fig. 3 Steady-state calibration curve

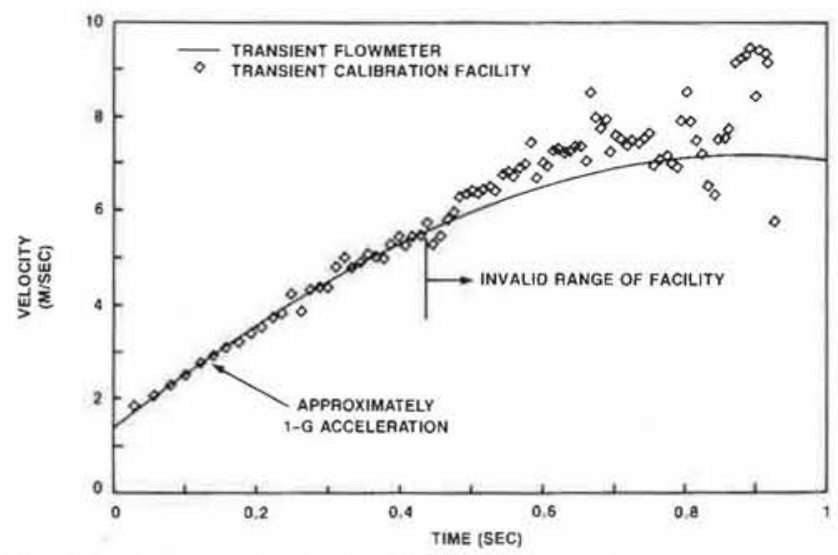

Fig. 4 Typical transient calibration curve (uncertainty in flow rate = \pm 5 percent, in time $= \pm 0.0002 \mathrm{sec}$

was established by draining the water at a very slow rate and weighing the amount of water collected between trigger pulses. The weight was converted into an effective distance between optical sensor pairs based on a constant 5 -cm internal pipe diameter, as shown in equation (2)

$$
L=W /(A \rho),
$$

where $\rho$ is the density of the water, $A$ is the cross-sectional area based on a $5 \mathrm{~cm}$ diameter, and $W$ is the weight of the water between optical sensor pairs. This method accounted for variations in vertical distance between optical sensors pairs and also variations in diameter along the pipe.

Following each calibration run, post-processing software scanned the raw data file and created a file of the time at which each optical sensor pair was triggered. Velocity, based on a 5$\mathrm{cm}$ diameter, was subsequently calculated from the time and effective distance values between optical pairs.

During a calibration, the analog output from the transient flowmeter was sampled simultaneously with the facility electronics at the $5000-\mathrm{Hz}$ sampling rate. Since the output of the flowmeter was updated at a rate of $60 \mathrm{~Hz}$, post-processing software scanned the raw data file and established the time corresponding to the updated flowmeter output to within \pm 0.0002 second. For each of these update times, the corresponding calibration velocity was calculated from the calibration velocity versus time data file by linearly interpolating between adjacent points. A comparison of the calibration velocity and the flowmeter velocity was then made.
Table 1 Calibration Summary

\begin{tabular}{ccr}
\hline Run number & Mean Error $(\%)$ & $\sigma$ \\
\hline 1 & 1.370 & 2.55 \\
2 & -0.946 & 2.56 \\
3 & -0.740 & 2.54 \\
4 & 0.276 & 2.67 \\
5 & -0.575 & 2.47 \\
6 & 0.776 & 2.62 \\
7 & -0.536 & 1.78 \\
8 & 0.378 & 2.88 \\
9 & 0.352 & 1.88 \\
10 & 0.807 & 3.33 \\
11 & -1.318 & 2.36 \\
12 & -1.191 & 2.88 \\
\hline Average for all runs: & -0.041 & 2.57 \\
\hline
\end{tabular}

\section{Results}

Prior to conducting any of the transient calibrations, a steadystate calibration of the meter was conducted at the Alden Research Laboratory's Flowmeter Calibration Facility, Holden, MA. This calibration was performed by using the gravimetric method and is considered accurate to \pm 0.25 percent. The calibration was conducted over a velocity range of 0.15 to $9.1 \mathrm{~m} / \mathrm{s}$ in the $5-\mathrm{cm}$ diameter flowmeter. The corresponding Reynolds number range was 7100 to 375,000 .

The steady-state calibration curve is shown in Fig. 3. The curve is presented as percent error from actual velocity when flowmeter velocity is calculated with a constant meter factor (K). As shown, accuracy (linearity) was within \pm 1.7 percent over the complete flow range and within \pm 0.5 percent over the 40,000 to 375,000 Reynolds number range, which is a substantial portion of the total range.

Twelve transient calibration runs were conducted. Even though each run was initiated by manually opening the valve at the bottom of the facility, the velocity versus time curve was fairly consistent between runs.

Results for a representative run are shown in the crosssectional averaged velocity versus time curve of Fig. 4 . The run lasted approximately 1 second. From 0.0 to approximately 0.43 second, the flow experienced an almost constant acceleration of approximately $1 \mathrm{~g}$, reaching a velocity of approximately $5.5 \mathrm{~m} / \mathrm{s}$. Beyond 0.43 second, the free surface of the water column experienced considerable instability precluding any accurate measurements. The flowmeter, however, appears to have followed the water column velocity throughout the transient. All further discussions are limited to the 0.0 -second to 0.43 -second range of each run, which corresponds to the first 29 data points from the flowmeter.

Results for all 12 of the calibration runs are presented in Table 1. Mean error and standard deviation between the calibration facility and flowmeter measured flow velocities are presented separately for each run and also for the total of all data points. As shown, the absolute value of the mean error ranged from 0.276 to 1.370 percent, while the standard deviation of the error ranged from 1.78 to 3.33 percent. The mean error for all the data points was -0.041 percent with a standard deviation of 2.57 percent.

Using the results from the total of all data points, the difference between the calibration facility and the flowmeter measurements is expected to be within \pm 5.2 percent for 95 percent of measurements to be taken.

Inspection of the transient calibration curves (similar to Fig. 4) for each of the twelve runs showed that the flowmeter output exhibited a smooth transition throughout the transient with negligible fluctuations about the mean curve. Furthermore, for a major portion of each individual run, the flowmeter curve is essentially the mean curve for the calibration facility data. 
Considering that the facility data for all runs fluctuated about the mean curves with a standard deviation of approximately 2.5 percent, it is believed that the accuracy (95 percent confidence level) of the facility is approximately \pm 5 percent. An error analysis showed that errors due to factors other than the data fluctuations were negligible relative to the 5 percent error due to the fluctuations.

Considering the above estimated facility accuracy, the flowmeter accuracy cannot be stated to be any better than the facility's \pm 5 percent accuracy during a transient run. However, considering that the mean difference between the facility and the flowmeter when all points are included is only -0.041 percent, and also considering the fact that the fluctuations in the flowmeter output about its mean curve are essentially negligible, it is expected that the true accuracy of the flowmeter in monitoring transient flows is approximately equal to the steady-state accuracy previously stated.

For the present meter, inaccuracies resulting from transient effects are essentially caused by the immediate rate of change of velocity profile and not its total past history. Therefore, even though the present tests were conducted with the fluid initially at rest, the above accuracy statements are applicable to other initial conditions.

\section{Conclusions}

An electromagnetic flowmeter has been developed that can accurately measure transient flow rates up to at least $1-\mathrm{g}$ acceleration (maximum tested) with a data rate to $60 \mathrm{~Hz}$. A calibration facility using a free-falling column of water has been shown to be a viable means of evaluating transient flowmeter performance up to $1 \mathrm{~g}$.

\section{Acknowledgment}

Support for this project was provided through the Independent Research Program at the Naval Underwater Systems Center, Newport, RI.

\section{References}

1 Arnold, J. S., "An Electromagnetic Flowmeter for Transient Flow Studies," The Review of Scientific Instruments, Vol. 22, No. 1, Jan. 1950.

2 Iwanicki, L. R., and Fontaine, R. J., "Application of an Electromagnetic Flowmeter in Unsteady Flow," ASME Symposium on Measurement in Unsteady Flow, May 1962.

3 Shercliff, J. A., The Theory of Electromagnetic Flow Measurement, Cambridge University Press, Cambridge, England, 1961.

4 Reinhold, I., "Velocity Profile Influence on Electromagnetic Flowmeter Accuracy," Flow Measurement of Fluids, North-Holland Publishing Co., 1978.

\section{Fluids Engineering Spring Conference}

The 1990 Spring Meeting of the Fluids Engineering Division will be held in conjunction with the Mechanical Engineering Forum of the Canadian Society of Mechanical Engineers at the University of Toronto, June 3-9, 1990.

The following FED symposia are planned.

- Nonsteady Viscous Flow

- Numerical Methods in Multiphase Flows

- Heuristics of Thermal Anemometry

The FED Forums include

- Flow Measurement and Instrumentation

- Cavitation and Multiphase Flow

- Fluid Machinery

- Turbulent Flows

- Bench Marks for Computational Fluid Mechanics

The CSME sessions being held in parallel include Advanced Manufacturing, Mechanics of Solids, Microgravity, Mechanical Engineering Education, Thermosciences, Bioengineering, Ship \& Offshore Dynamics, and Micro-Mechanics.

For further information on the FED program, contact Prof. C. T. Crowe, Dept. of Mech. and Matl. Engr., Washington State Univ., Pullman, WA 99164-2920 (509-335-3214). Information on the CSME Forum and general conference information can be obtained from Prof. F. P. J. Rimrott, Dept. of Mech. Engr., University of Toronto, Toronto, Ont., CANADA M5S 1A4 (416-9783053). 